\title{
On the optimal energy efficiency and spectral efficiency trade-off of CF massive MIMO SWIPT system
}

\author{
Na Li ${ }^{*}$, Yuanyuan Gao and Kui Xu
}

\author{
*Correspondence: \\ linalala@yeah.net \\ Army Engineering University \\ of PLA, Nanjing 210007, \\ People's Republic of China
}

\begin{abstract}
Energy efficiency is a key requirement for future network design, and user-centric (UC) cell-free (CF) massive multi-input multi-output (MIMO) networks can achieve over ten times the energy efficiency. Based on this, this paper studies a CF MIMO simultaneous wireless information and power transmission system and proposes a UC access point (AP) selection method and a trade-off performance optimization scheme for spectral efficiency and energy efficiency. In this system, users have both energy recovery and information transmission functions. According to the difference between the interference in the energy harvesting and information transmission process, a flexible AP selection scheme is designed. Blindly pursuing high spectral efficiency will result in waste of resources. This paper proposes an evaluation index that takes into account both energy efficiency and spectral efficiency, analyses the trade-off between energy efficiency and spectral efficiency, and jointly optimizes the AP selection scheme and the uplink (UL) and downlink (DL) time switching ratio to maximize the trade-off performance. Then, the non-convex problem is converted to a geometric planning problem to solve. The simulation results show that by implementing a suitable AP selection scheme and UL and DL time allocation, the information processing scheme on the AP side has a slight loss in spectral efficiency, but the energy efficiency is close to the performance of global processing on the central processing unit.
\end{abstract}

Keywords: Cell-free, Simultaneous wireless information and power transmission, Usercentric, Energy efficiency, Spectral efficiency, Trade-off optimization

\section{Introduction}

The SEBy equipping base stations with massive multiple-input multiple-output (MIMO) networks, the spectral efficiency (SE) can be increased by at least 10 times compared with traditional cellular networks [1-3]. The antennas in traditional massive MIMO systems are deployed in a centralized manner, which can improve the performance of the network. At the same time, this method faces problems such as poor communication quality, large path loss, unsatisfactory network coverage, low energy efficiency (EE), complex channel estimation, and great mutual interference between cells at the edge [4]. A cell-free (CF) massive MIMO system addresses the above-mentioned series of problems by placing a large number of single-antenna or multi-antenna access points (APs)

c) The Author(s), 2021. Open Access This article is licensed under a Creative Commons Attribution 4.0 International License, which permits use, sharing, adaptation, distribution and reproduction in any medium or format, as long as you give appropriate credit to the original author(s) and the source, provide a link to the Creative Commons licence, and indicate if changes were made. The images or other third party material in this article are included in the article's Creative Commons licence, unless indicated otherwise in a credit line to the material. If material is not included in the article's Creative Commons licence and your intended use is not permitted by statutory regulation or exceeds the permitted use, you will need to obtain permission directly from the copyright holder. To view a copy of this licence, visit http:// creativecommons.org/licenses/by/4.0/. 
in a certain geographic location [5-7]. In the CF massive MIMO architecture, all APs are connected to a central processing unit (CPU) through a backhaul link, and the CPU is responsible for coordinating various resource allocations. Because of the user-centric (UC) cooperation relationship between APs, the cell boundary is eliminated to a certain extent, which avoids the influence of cell interference. In addition, the distance between the APs and the user is smaller than that in a traditional cellular network, which leads to lower path loss and higher diversity gain [8-10].

With the development of the Internet of Things (IoT) [11] and large-scale links, wireless energy transmission technology has increasingly demonstrated its importance [12, 13]. Since most IoT devices are battery powered, it is difficult for these devices to maintain communication with limited energy. SWIPT technology can provide energy for wireless devices to extend their working hours without significantly increasing the configuration of foundational equipment. SWIPT technology has undergone much research in massive MIMO, and research in CF massive MIMO has just started. In fact, in CF massive MIMO systems, high-density APs are more conducive to realizing SWIPT [14, 15]. The realization of SWIPT comes at the cost of decreased throughput [15-17], and the process of energy harvesting will affect the SE of information transmission in a system. In a CF massive MIMO system, all APs are distributed in the entire coverage area to serve all users at the same frequency, which leads to the problems that some APs are far away from some users and that the path loss between them is inevitably large. Reference [18] adopted a UC approach, in which each user has only some of the APs to provide services. A simulation showed that the UC method can increase the user's total achievable rate and reduce the backhaul overhead. The combination of SWIPT technology and UC CF massive MIMO can extend the communication time and compensate for lost throughput. Therefore, the combination of SWIPT and CF massive MIMO will achieve new vitality in future $5 \mathrm{G}$ and higher versions.

In the past ten years, in the field of massive MIMO, to improve throughput, researchers have made much effort, for example, by optimizing the number of base stations (BSs) and antennas in a cellular network [19], using relay cooperative transmission [20], and optimizing beamforming [21] and resource allocation [22]. However, high network throughput usually means considerable energy consumption, which is not feasible for energy-constrained devices. For a CF massive MIMO network, increasing the total number of APs and the number of antennas per AP can improve the SE of the system [23], but at the same time, it will further increase the backhaul load, which is the main challenge of the distributed antenna system [24]. Therefore, reducing energy consumption while meeting the SE requirements of such networks and devices is a top priority. With the increase in coverage and user access, global telecommunication networks (including wired, wireless and core networks) account for more than one-third of energy consumption [25]. In this context, EE has gradually become an important standard in the design of communication networks. In future mobile systems, while maintaining a certain SE, energy consumption must be considered to reduce the ever-increasing operating costs of the network [26, 27]. However, to maximize the SE, it is necessary to minimize the unit energy consumption, so the EE and SE form a pair of contradictory goals. Therefore, the trade-off between the SE and EE becomes the main criterion for designing efficient communication systems [28]. Compared with centralized massive MIMO, a cell-free massive 
MIMO system can provide very high EE [29], so it is more meaningful to study SE and EE trade-offs in CF massive MIMO architectures.

\subsection{Previous works}

There is extensive research about SWIPT in massive MIMO. SWIPT technology has two basic architectures: time switching and power distribution. Reference [30] studied a three-dimensional massive MIMO SWIPT system, where the approximate expressions of SINR and harvested energy are derived and Under the constraints of SINR and received power, the antenna inclination and power allocation factor are jointly optimized to minimize the transmit power. In paper [31], an effective resource allocation scheme is proposed for multiuser massive MIMO SWIPT system with imperfect channel estimation. The optimization of system energy efficiency is proposed from the aspects of beamforming design, antenna selection, power allocation and time division protocol, through simulation, the effectiveness of the proposed scheme and the performance superior to the existing scheme are proved. Reference [32] considers the trade-off of single-user massive MIMO SE-EE. Reference [33] studies the SE-EE trade-off in generalized spatial modulation schemes in multi-user massive MIMO systems. The results show that the spatial modulation with only one active antenna per user can provide less SE, but it is the most energy-saving transmission mode, and the EE of spatial modulation is better than that of traditional massive MIMO schemes. Reference [34] uses the max-min fairness optimal transmission power control strategy in the massive MIMO SWIPT system to derive the optimal rate-energy balance to ensure user fairness. Reference [35] studies the trade-off problem of SE-EE in beam-domain massive MIMO. Reference [36] studies the trade-off between SE and EE in centralized, distributed, and hybrid massive MIMO. The simulation results show that the EE of distributed massive MIMO is higher than that of other massive MIMO when the number of antennas is constant.

Due to the inherent path loss, research on SWIPT technology in centralized massive MIMO has not been able to overcome this bottleneck. In a CF MIMO network, APs can be randomly distributed, which reduces the distance between APs and users and improves the efficiency of energy harvesting. Therefore, some works have begun to consider CF massive MIMO SWIPT systems, which use distributed APs to charge and transmit information to users in the coverage area.

References [37-39] studied CF massive MIMO SWIPT systems. Reference [37] researches the performance of a CF massive MIMO SWIPT system in which information and power are simultaneously transmitted to single-antenna information or energy users through a large number of spatially distributed single-antenna APs. Then, the closed-form expressions of the recovered energy and DL/UL achievable rate are deduced. The simulation results show that CF massive MIMO can improve the performance of SWIPT by taking advantage of distributed transmission and reception with a large number of APs. Reference [38] considers a spatial stochastic network in which the Poisson point process is used to model APs randomly and the time switch protocol is used for SWIPT operation at the user. It also considers blockages caused by obstacles in the channel and line-of-sight and non-line-of-sight conditions that affect fading and path loss. Then, the average energy and variance of energy users and the average achievable rate in the DL are obtained. The trade-off between the DL 
data throughput and collected energy is quantified, and it is shown that in a CF massive MIMO configuration, APs distributed in space can improve the trade-off between SWIPT energy and throughput. Reference [39] studies the energy-saving resource allocation of non-orthogonal multicast and unicast transmission based on layered division multiplexing in a CF massive MIMO SWIPT system. Then, a non-smooth, non-convex optimization problem is proposed for maximizing EE, and a first-order algorithm is developed to find the initial feasible solution.

The above research on the CF massive MIMO SWIPT system considers different distribution models, transmission models, and energy harvesting models, but without considering the demand to mobilize all AP services for users, it does not give full play to the advantages of the distributed antenna, resulting in a waste of resources. Previous works lack research on the trade-off between EE and SE and how to quantitatively evaluate the trade-off performance, which will be an increasingly important field in the future.

\subsection{Contributions}

In order to fill the above gaps, we studied the trade-off between EE and SE of the CF massive MIMO SWIPT system, and proposed an evaluation index that takes both SE and EE into consideration. This determines the implementation of AP selection schemes to optimize system performance. The specific contributions of this paper are as follows:

(1) The SE-EE trade-off of CF massive MIMO SWIPT system is studied for the first time. Since SE and EE are a pair of contradictory indicators, the trade-off of SE and $\mathrm{EE}$ is a key issue in system design. Considering that $\mathrm{CF}$ gains the user's achievable rate at the cost of the backhaul link load, this article puts the information processing capability on the AP, and the $\mathrm{CPU}$ only performs data transmission. The closedform expressions for the harvested energy and the achievable UL and DL rates are derived, and in this scenario, the compromise performance of SE-EE is studied.

(2) In actual transmission, APs that are far away from the user contribute less to the SE of the user in the communication process, because they mainly cause strong interference. In the UC method, only some APs provide services to users, which can improve system EE and reduce backhaul load. In the CF massive MIMO SWIPT system proposed in this article, according to the different characteristics of energy harvesting and information transmission, a flexible AP selection scheme is designed to achieve the SE and EE trade-off.

(3) Aiming at the SE and EE trade-off, a trade-off performance index that takes into account system SE and EE is proposed, and the SWIPT time switching parameters and the number of service antennas in UC mode are jointly optimized to maximize the trade-off performance of the system. The new optimization problem brought about by distributed antenna, the non-convex problem is rewritten into a convex problem using approximate formulas, and geometric planning (GP) is used to solve it. The optimization simulation results show that after optimization, The solution proposed in this paper for data processing on the AP has a much higher energy efficiency than the centralized processing on the CPU. 
Notation Bold indicates vectors; $x \sim \mathbb{C N}\left(0, \sigma^{2}\right)$ means that $x$ is a complex Gaussian random variable with zero mean and a variance of $\sigma^{2} ;()^{T},()^{H}$ represent the transpose and conjugate transpose, respectively; and \|\|$, \mathbb{E}($ ) represent the Euclidean norm and mathematical expectation, respectively.

The rest of this paper is organized as follows: Sect. 2 presents the system model. Section 3 presents the SE and EE performance analysis. Section 4 presents the simulation results. Section 5 draws conclusions.

\section{System model}

Consider a UC CF massive MIMO SWIPT system in which M APs and K users are randomly distributed in the coverage area and all APs connect to a CPU that performs data decoding through a backhaul link. All communications are carried out on the same frequency band and work in time division duplex (TDD) mode. Based on the channel reciprocity of the TDD system and the channel hardening due to multiple antennas [40], only UL channel estimation is performed. The UC CF massive MIMO system model diagram is shown in Fig. 1. Each AP sends and receives data with only some specific users.

Both APs and users are equipped with a single antenna. The channel matrix between the $m$-th AP and the $k$-th user is expressed as $g_{m, k}=\sqrt{\beta_{m, k}} h_{m, k}$, where $\beta_{m, k}$ is the large-scale fading factor, $h_{m, k}$ represents the small-scale fading, and the small-scale fading elements obey a complex Gaussian distribution $\mathbb{C N}(0,1)$. According to reference [30], large-scale fading is modelled as $\beta_{m, k}=10^{\frac{\mathrm{PL}_{m . k}}{10}} 10^{\frac{\sigma_{m, k} v_{m . k}}{10}}$, where $\sigma_{m, k} v_{m, k}$ is the shadow fading coefficient between AP $\mathrm{m}$ and user $\mathrm{k}$ and the path loss coefficient between AP $\mathrm{m}$ and user $\mathrm{k}$ is expressed as follows:

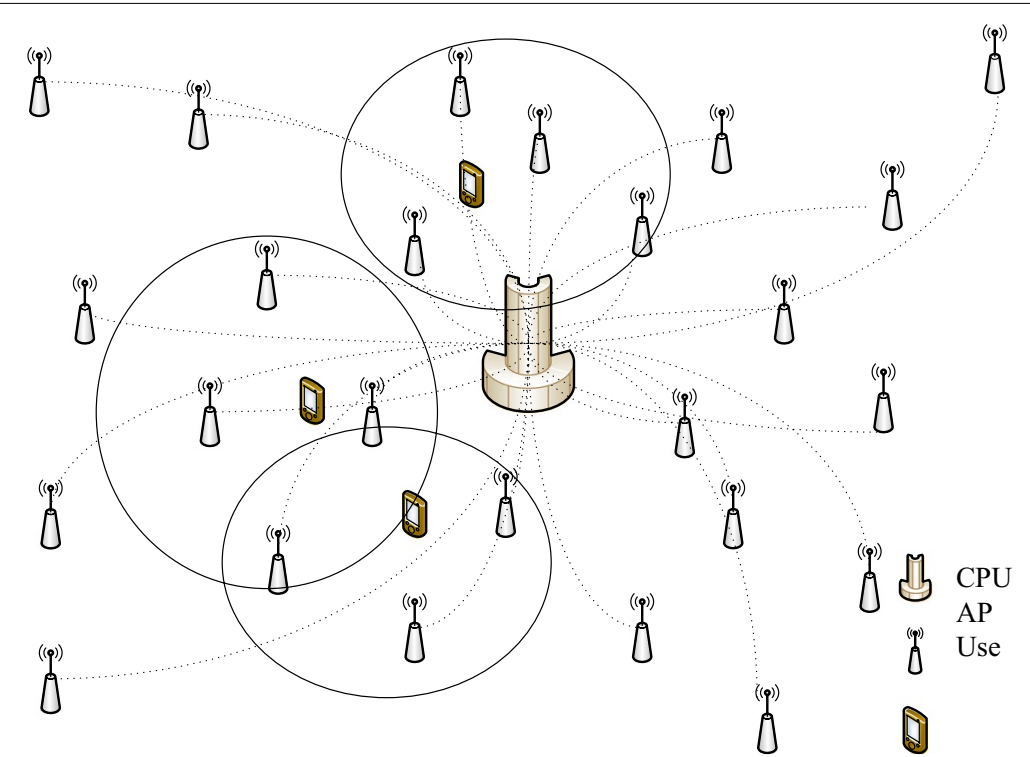

Fig. 1 UC CF massive MIMO system 


$$
\mathrm{PL}_{m, k}=\left\{\begin{array}{l}
-L-35 \log _{10}\left(d_{m, k}\right), d_{m, k}>d_{1} \\
-L-35 \log _{10}\left(d_{1}^{1.5} d_{m, k}^{2}\right), d_{0}<d_{m, k} \leqslant d_{1} \\
-L-35 \log _{10}\left(d_{1}^{1.5} d_{0}^{2}\right), d_{0}<d_{m, k}
\end{array}\right.
$$

where

$$
\begin{aligned}
L= & 46.3+33.9 \log (G)-13.82 \log \left(h_{A P}\right) \\
& -(1.11 \log (G)-0.7) \mathrm{h}_{\mathrm{K}}+1.56 \log (G)-0.8
\end{aligned}
$$

This paper selects the parameter settings of the medium-sized city and suburban model, where $G$ is the centre frequency, $\mathrm{h}_{\mathrm{AP}}$ and $\mathrm{h}_{\mathrm{K}}$ represent the heights of the AP and user k, respectively, $d_{0}$ and $d_{1}$ are reference distances, $d_{m, k}$ is the distance between AP $\mathrm{m}$ and user $\mathrm{k}$, and the shadow fading model is as follows:

$$
v_{m, k}=\sqrt{\eta} a_{m}+\sqrt{1-\eta} b_{m}
$$

where the elements of $a_{m}, b_{m}$ obey a complex Gaussian distribution with mean zero and variance one. Variables $a_{m}$ and $b_{m}$ respectively model the shadow fading caused by obstacles near the $\mathrm{m}$-th AP and the k-th user. $\eta$ is random parameter between 0 and 1. The parameter $\eta$ depends on the influence of the AP and obstacles around the user on the shadow fading. The covariance matrices are $E\left[a_{m} a_{m^{\prime}}\right]=2^{-\frac{d_{A P\left(m, m^{\prime}\right)}}{d_{e}}}$ and $E\left[b_{k} b_{k^{\prime}}\right]=2^{-\frac{d_{K\left(k, k^{\prime}\right)}}{d_{e}}}$, respectively, where $d_{A P\left(m, m^{\prime}\right)}$ is the geographic distance between two APs, $d_{K\left(k, k^{\prime}\right)}$ is the geographic distance between two users, $d_{e}$ is a fixed value related to the environment, and there is no shadow fading when $d_{m, k} \leqslant d_{1}$.

The whole transmission process based on the frame is divided into three stages. First, the user sends pilots to the AP for UL training. Second, the AP uses the estimated channel state information (CSI) for DL information transmission and DL energy harvesting, and finally, based on channel reciprocity, the user uses the harvested energy to send information to the AP. The transmission model and flow chart are shown in Figs. 2 and 3.

\subsection{Channel estimation}

In the channel estimation stage, $\mathrm{K}$ users send pilot signals $\mathbf{S}=\left[\mathbf{s}_{1}, \ldots, \mathbf{s}_{k}, \ldots, \mathbf{s}_{K}\right] \in \mathbb{C}^{K \times \tau_{P}}$ to all APs at the same time, where $\mathbf{s}_{k} \in \mathbb{C}^{1 \times \tau_{P}}$ is the pilot signal sent by user $\mathrm{k}$ to $\mathrm{m}$ APs,

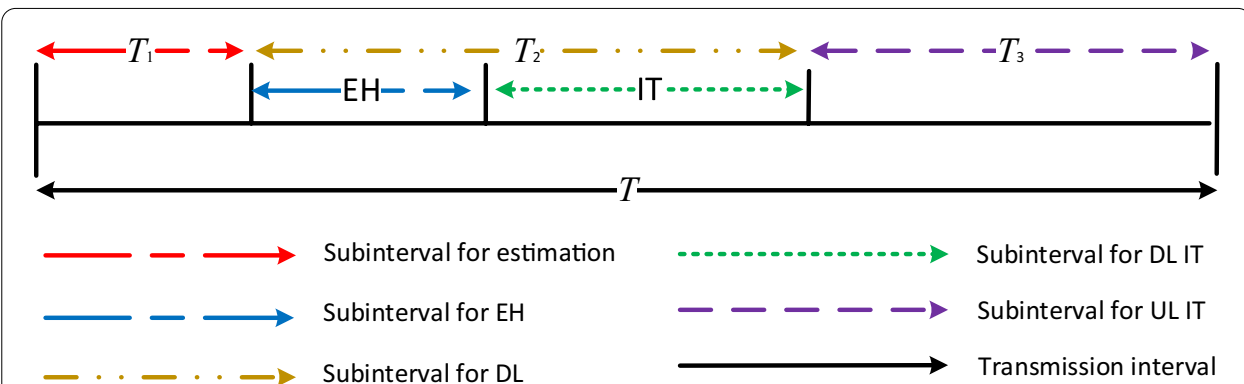

Fig. 2 Transmission interval model 


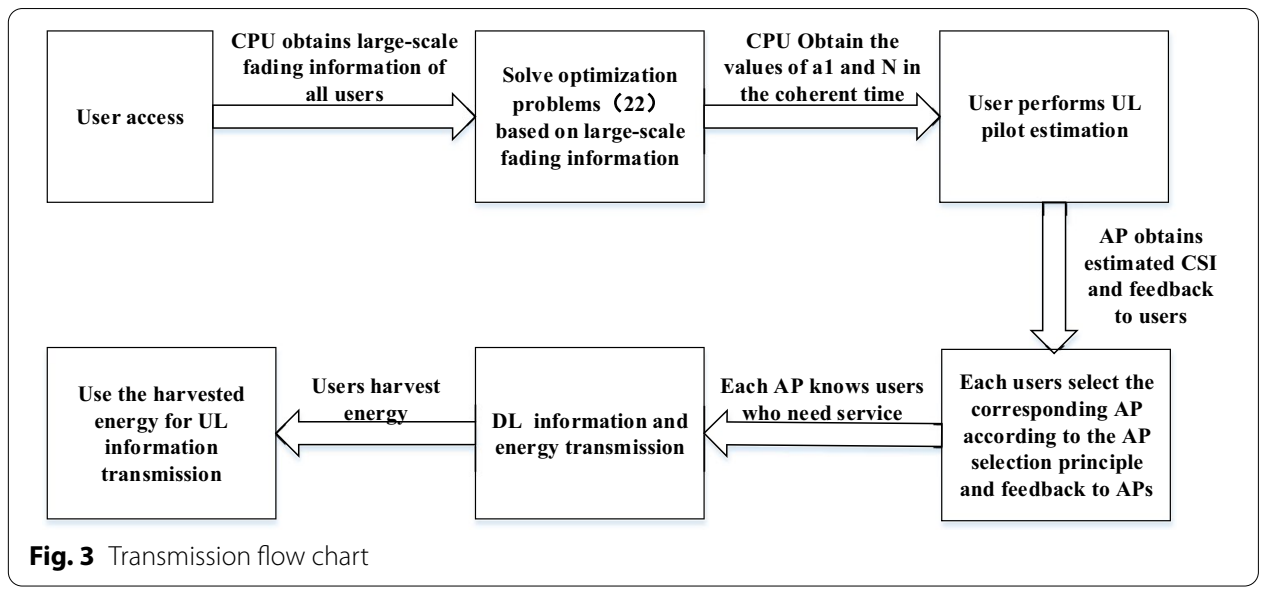

$\tau_{P}$ is the pilot length, the K pilot sequences are orthogonal, and $\left\|\mathbf{s}_{k}\right\|^{2}=1$. Then, the pilot signal received by the $\mathrm{m}$-th $\mathrm{AP}$ is

$$
\mathbf{y}_{m}=\sum_{k=1}^{K} \sqrt{\tau_{p} P_{p}} g_{m, k} \mathbf{s}_{k}+\mathbf{N}_{m}
$$

where $P_{p}$ is the pilot power, $\mathbf{N}_{m} \in \mathbb{C}^{1 \times \tau_{P}}$ is the noise vector, and its elements satisfy the independent and identically distributed complex Gaussian distribution $\mathbb{C N}\left(0, \sigma^{2}\right)$, where $\sigma^{2}$ is the noise power.

The Minimum mean square error (MMSE) [31] method is used to estimate the channel, which performs correlation processing on the pilot received at the AP.

$$
\begin{aligned}
\hat{y}_{m, k} & =\mathbf{y}_{m} \mathbf{s}_{k}^{H} \\
& =\left(\sum_{k=1}^{K} \sqrt{\tau_{p} P_{p}} g_{m, k} \mathbf{s}_{k}+\mathbf{N}_{m}\right) \mathbf{s}_{k}^{H} \\
& =\sqrt{\tau_{p} P_{p}} g_{m, k}+\mathbf{N}_{m} \mathbf{s}_{k}^{H}
\end{aligned}
$$

Therefore, the estimated channel is

$$
\begin{aligned}
\hat{g}_{m, k} & =\frac{\mathrm{E}\left\{\hat{y}_{m, k}^{H} g_{m, k}\right\}}{\mathrm{E}\left\{\left|\hat{y}_{m, k}\right|^{2}\right\}} \hat{y}_{m, k} \\
& =c_{m, k}\left(\sqrt{\tau_{p} P_{p}} g_{m, k}+\mathbf{N}_{m} \mathbf{s}_{k}^{H}\right)
\end{aligned}
$$

where

$$
c_{m, k}=\frac{\sqrt{\tau_{p} P_{p}} \beta_{m, k}}{\tau_{p} P_{p} \beta_{m, k}+\sigma^{2}}
$$




\subsection{AP selection}

The contribution of the APs far from the user can be ignored in the transmission; therefore, it is not necessary that all APs provide service to a certain user. The UC method selects some APs close to the user to serve her. The UC method can reduce interference between users and increase the achievable rate per user.

This article uses maximal ratio combining (MRC) for precoding and detection on APs, which can effectively reduce the backhaul load and the working pressure of the CPU. In this case, for user information transmission, inter-user interference should be minimized to improve the SE of the system. However, for energy recovery, inter-user interference is beneficial and can increase the energy recovered by users, so different AP selection schemes should be used in the energy recovery and information transmission stages.

In the energy harvesting stage, the total energy harvested increases with the increase in the number of service APs. Although the performance of using MRC precoding at the AP is not as good as the performance of using zero-forcing (ZF) precoding at the $\mathrm{CPU}$, it can significantly reduce the CPU load. Therefore, in the energy harvesting stage, no AP selection is performed, which means that all APs serve $\mathrm{K}$ users at the same time. According to Fig. 4, when MRC is used at the AP, as the number of service APs increases, the user's achievable rate tends to a stable value. Therefore, AP selection during the information transmission stage can reduce hardware loss while maintaining system performance.

AP selection principle: according to the channel state information, the users served by AP $\mathrm{m}$ are $\kappa(m) \in\{1, \ldots, K\}$, and AP $\mathrm{m}$ serves only the $\mathrm{N}$ users with the largest Frobenius norm of its channel $\kappa(m)=\max _{N}\left\|\hat{g}_{k}\right\|_{\mathrm{F}}$, where $\hat{g}_{k}$ is the estimated channel between user $k$ and all APs.

The process of AP selection in this article is as follows: in the channel estimation stage, the users send pilot signals to the APs, each AP obtains CSI for all users and feeds back the CSI to the users, and each user selects the user who serves him according to the principle of AP selection and informs the AP that he needs to provide services for himself. During the DL energy transmission phase, no AP selection is performed. When the energy transmission is completed and the DL information transmission phase is entered, the AP allocates power to the users that need to be served according to the information fed back by the users, and the AP selection time is ignored relative to the coherence time.

The AP selection method adopted in this paper is as follows: in the channel estimation stage, the users send pilot signals to the APs, and each AP obtains CSI for all users. During the DL energy transmission phase, no AP selection is performed. When the energy harvesting is completed, the user enters the information receiving stage, the AP selects the users it serves, and the AP selection time is ignored relative to the coherent time.

\subsection{Downlink energy and information transmission}

After channel estimation, the AP has the CSI of all the users, and AP m selects the users that need to be served according to the different requirements of energy and information $\kappa(m) \in\{1, \ldots, K\}$ and sends information to the selected users based on the CSI. The DL signal sent by AP $\mathrm{m}$ to user $\mathrm{k}$ is $x_{m}=\sum_{k \in \kappa(m)} \sqrt{\vartheta_{m, k} P_{t}} w_{m, k} q_{k}$, where $q_{k}$ 
represents the energy signal sent to user $\mathrm{k}, P_{t}$ is the transmission power of AP $\mathrm{m}, \vartheta_{m, k}$ represents the power allocation parameter of the signal sent by AP $\mathrm{m}$ to user $\mathrm{k}$, and $\mathbf{W}_{m}=\left[w_{m, 1}, \ldots w_{m, k}, \ldots w_{m, K}\right]$ is the signal precoding vector. In this article, to reduce the pressure on the CPU, the CPU transmits information only to the AP, and the information is processed in the AP. DL precoding uses the maximum ratio transmission (MRT) scheme $\mathbf{W}_{m}=\alpha_{m} \mathbf{G}_{m}$, where $\alpha_{m}$ is the power normalization coefficient of $\mathrm{AP} \mathrm{m}$ and meets the power limit

$$
\begin{aligned}
& \mathbb{E}\left\{\left|x_{m}\right|^{2}\right\} P_{t} \\
& \Rightarrow \alpha_{m}=\sqrt{1 / \sum_{k \in \kappa(m)} \vartheta_{m, k} \Upsilon_{m, k}}
\end{aligned}
$$

where

$$
\Upsilon_{m, k}=\mathbb{E}\left\{\left|\hat{g}_{m, k}\right|^{2}\right\}=\frac{\left(\sqrt{\tau_{p} P_{P}} \beta_{m, k}\right)^{2}}{\tau_{p} P_{P} \beta_{m, k}+\sigma^{2}}
$$

Due to the particularity of energy harvesting, inter-user interference will increase the energy harvesting efficiency. Then, the signal received by user $k$ is

$$
y_{d, k}=\sum_{m=1}^{M} g_{m, k} \sum_{k^{\prime} \in \kappa(m)} \sqrt{\vartheta_{m, k^{\prime}} P_{t}} w_{m, k^{\prime}} q_{k^{\prime}}+n_{d, k}
$$

Since the noise power is much smaller than the power of the energy signal, the influence of noise can be ignored during the transmission process; then, the energy received by user $\mathrm{k}$ is

$$
E_{k}=\eta \tau_{e}\left\|\sum_{m=1}^{M} \sum_{k^{\prime}=1}^{K} g_{m, k} \sqrt{\vartheta_{m, k^{\prime}} P_{t}} w_{m, k^{\prime}} q_{k^{\prime}}\right\|^{2}
$$

where $\eta$ is the energy conversion efficiency. Then, the user switches to the information transmission mode. To analyse the DL transmission, formula (9) can be rewritten as follows:

$$
\begin{aligned}
y_{d, k}= & \underbrace{\mathbb{E}\left\{\sum_{m=1}^{M} g_{m, k} \sqrt{\vartheta_{m, k} P_{t}} w_{m, k}\right\}}_{\mathrm{F}_{1}} q_{k} \\
& +\underbrace{\left(\sum_{m=1}^{M} g_{m, k} \sqrt{\vartheta_{m, k} P_{t}} w_{m, k}-\mathbb{E}\left\{\sum_{m=1}^{M} g_{m, k} \sqrt{\vartheta_{m, k} P_{t}} w_{m, k}\right\}\right) q_{k}}_{F_{2}} \\
& +\underbrace{\sum_{k^{\prime} \in \kappa(m), k^{\prime} \neq k} \sum_{m=1}^{M} g_{m, k} \sqrt{\vartheta_{m, k^{\prime}} P_{t}} w_{m, k^{\prime}} q_{k^{\prime}}}_{\mathrm{F}_{3}}+n_{d, k}
\end{aligned}
$$


where $F_{1}$ is the desired signal of user $k, F_{2}$ is the precoding uncertainty, and $F_{3}$ is the interference between users. According to the independence of the signal, channel and noise, the signal-to-interference-plus-noise ratio (SINR) of DL channel transmission can be obtained as

$$
\gamma_{d, k}=\frac{\left|\sum_{m=1}^{M} \alpha_{m} \sqrt{\vartheta_{m, k} P_{t}} \Upsilon_{m, k}\right|^{2}}{\sum_{k^{\prime} \in \kappa(m)}\left(\sum_{m=1}^{M} \alpha_{m}^{2} P_{t} \beta_{m, k} \vartheta_{m, k^{\prime}} \Upsilon_{m, k^{\prime}}\right)+\sigma^{2}}
$$

Then, the users send information $\Phi=\left[\phi_{1}, \ldots, \phi_{K}\right]$ to the AP, where $\phi_{k}$ is the information sent by user $k$ to all APs; for AP $m$, the received information is

$$
y_{m}=\sum_{k \in \kappa(m)} \sqrt{\psi_{k}} g_{m, k} \phi_{k}+n_{m}
$$

where $\psi_{k}=\left\|\phi_{k}\right\|^{2}=\frac{\eta \tau_{e}}{\tau_{u}}\left\|\sum_{m=1}^{M} \sum_{k^{\prime}=1}^{K} g_{m, k} \sqrt{\vartheta_{m, k^{\prime}}} w_{m, k^{\prime}} q_{k^{\prime}}\right\|^{2}$.

The feedback time is ignored. Similar to the derivation of the DL, the information processing is performed by the AP. AP m processes the information through the corresponding receiving matrix and sends the processed information to the CPU. Then, the total information received by the CPU. Then the total information received by the CPU during the UL information is

$$
\begin{aligned}
y_{u, k}= & \sum_{m \in \bar{M}(k)} w_{m, k}^{u} g_{m, k} \phi_{k} \\
& +\sum_{m \in \bar{M}(k)} \sum_{k \in \kappa(m), k \neq K} w_{m, k}^{u} g_{m, k} \phi_{k^{\prime}}+w_{m, k}^{u} n_{u}
\end{aligned}
$$

The SINR of user $k$ at the receiving end is

$$
\gamma_{u, k}=\frac{\psi_{k, u}\left|\sum_{m=1}^{M} \Upsilon_{m, k}\right|^{2}}{\sum_{k^{\prime} \in \kappa(m)}\left(\sum_{m=1}^{M} \beta_{m, k^{\prime}} \psi_{k^{\prime}, u} \Upsilon_{m, k}\right)+\sigma^{2} \sum_{m=1}^{M} \Upsilon_{m, k}}
$$

The formula derivation is shown in "Appendix A", and the derivation process refers to DL transmission.

\section{Performance analysis and optimization}

\subsection{Spectral efficiency and energy efficiency}

According to formulas (12) and (15), the SE is expressed as follows:

$$
\begin{aligned}
& \mathrm{SE}_{d, k}=\left(\frac{\tau_{d}}{T}\right) \log _{2}\left(1+\gamma_{d, k}\right) \\
& \mathrm{SE}_{u, k}=\left(\frac{\tau_{u}}{T}\right) \log _{2}\left(1+\gamma_{u, k}\right)
\end{aligned}
$$

According to reference [18], the EE model is as follows: 


$$
E_{e}=\frac{B \times S e}{p_{\text {total }}}
$$

where $B$ is the channel bandwidth, $S_{e}=\mathrm{SE}_{\mathrm{d}, \mathrm{k}}+\mathrm{SE}_{\mathrm{u}, \mathrm{k}}$ represents the total SE of the UL and DL transmissions of the $\mathrm{K}$ users, and $p_{\text {total }}$ indicates the total power consumption of the system, which is as follows:

$$
P_{\text {total }}=\sum_{m=1}^{M} P_{m}+\sum_{m=1}^{M} P_{b h, m}
$$

where $P_{m}$ indicates the amplifier and circuit power consumption,

$$
P_{m}=\frac{1}{a}\left|x_{m}\right|^{2}+P_{c, m}=\frac{1}{a}\left|\sum_{k=1}^{K} \sqrt{\vartheta_{m, k} P_{t}} w_{m, k}\right|^{2}+P_{c, m}
$$

where $a$ indicates the efficiency of the power amplifier and $P_{c, m}$ is the power consumption required to run the circuit components. $P_{b h, m}=p_{\bar{m}}+B \times S_{e} \times P_{b t, m}$ represents the power consumption of the backhaul link between the AP and CPU, where $p_{\bar{m}}$ represents the fixed power consumption of the backhaul, which depends on the distance between the AP and the CPU and the system topology, and $P_{b t, m}$ is the power consumption related to throughput (unit W/bit/s), according to formulas (17)-(19).

$$
E_{e}=\frac{B \times \mathrm{SE}}{\sum_{m=1}^{M}\left(p_{\bar{m}}+P_{c, m}+B\left(P_{b t, m}\right) \mathrm{SE}\right)+\frac{1}{a} \sum_{m=1}^{M} \sum_{k \in \kappa(m)} P_{t} \vartheta_{m, k} \Upsilon_{m, k}}
$$

In the UC CF massive MIMO SWIPT system, the above formula can be rewritten as

$$
E_{e}^{u c}=\frac{B \times \mathrm{SE}}{N\left(p_{\bar{m}}+P_{c, m}+B\left(P_{b t, m}\right) \mathrm{SE}\right)+\frac{1}{a} \sum_{m=1}^{M} \sum_{k \in \kappa(m)} P_{t} \vartheta_{m, k} \Upsilon_{m, k}}
$$

\subsection{Optimizing the time-switching parameters and number of service APs}

This section optimizes the trade-off of the SE and EE of the system. From Eqs. (16) and (21), the system SE increases with the number of service APs, but the EE behaves in the opposite way. In actual transmission, it is unreasonable to blindly pursue SE. High SE will bring about high energy consumption, and the requirements for SE in some transmission situations are not very high. In this case, the EE of the system must be considered. Therefore, this paper proposes a trade-off index that considers both SE and EE: $\Gamma=S_{e}{ }^{w_{s}} E_{e}{ }^{w_{e}}$, where $w_{s}$ and $w_{e}$ are weighting factors.

From formula (10), it can be seen that the longer the user's energy recovery time is, the greater the power used to send the UL signal, but the time used for information transmission and the SE decrease at the same time. The energy harvesting process of the SWIPT system will reduce the SE, so the smaller $\alpha_{2}$ is, the greater the SE of the system, but SWIPT technology can extend the user's standby time, assist the user in the 
transmission of UL information and achieve information interaction. During optimization, $\alpha_{2}$ is set to a fixed value.

When $\alpha_{2}$ is fixed, the larger $\alpha_{1}$ is, the more energy can be harvested, and the higher the power for uplink transmission for users, but the shorter the corresponding uplink user transmission time. When $N$ is larger, the power provided to users is larger, and the spectral efficiency of the system is higher, but at the same time, more system energy will be consumed. Therefore, the system performance can be optimized by jointly optimizing $N$ and $\alpha_{1}$.

$$
\begin{aligned}
& \max _{\alpha_{1}, N} S_{e}^{w_{s}} E_{e}^{w_{e}} \\
& \left\{\begin{array}{l}
c 1: \gamma_{u, k} \frac{\eta\left(\alpha_{1} \alpha_{2}\right)}{\left(1-\alpha_{1}\right)} \psi_{k} b_{u, k}\left(c_{u, k}+d_{u, k}\right)^{-1}, \quad k=1, \ldots, K \\
c 2: \gamma_{d, k} e_{d, k} / f_{d, k}, \quad k=1, \ldots, K \\
c 3: \sum_{k=1}^{K} \vartheta_{m, k} P_{d}, m=1, \ldots, M \\
c 4: 0 \alpha_{1} 1
\end{array}\right.
\end{aligned}
$$

where

$$
S_{e}=\xi_{1} \alpha_{1} \sum_{k=1}^{K} \log _{2}\left(1+\gamma_{d, k}\right)+\xi_{2}\left(1-\alpha_{1}\right) \sum_{k=1}^{K} \log _{2}\left(1+\gamma_{u, k}\right)
$$

where

$$
\psi_{k}=\frac{\eta \tau_{E}}{\tau_{u}} \tau_{p} P_{t} \sum_{m=1}^{M} \beta_{m, k} \sum_{k^{\prime}=1}^{K} \vartheta_{m, k^{\prime}} c_{m, k^{\prime}}^{2} \beta_{m, k^{\prime}}, b_{u, k}=\left(\sum_{m=1}^{M} \Upsilon_{m, k}\right)^{2}, c_{u, k}=\sum_{k^{\prime} \in \kappa\left(m_{2}\right)}\left(\sum_{m=1}^{M} \beta_{m, k^{\prime}} \psi_{k^{\prime}, u} \Upsilon_{m, k}\right)
$$

$, d_{u, k}=\sigma^{2} \sum_{m=1}^{M} \Upsilon_{m, k}, f_{d, k}=\sum_{k^{\prime} \in \kappa(m)}\left(\sum_{m=1}^{M} \alpha_{m}^{2} P_{t} \beta_{m, k} \vartheta_{m, k^{\prime}} \Upsilon_{m, k^{\prime}}\right)+\sigma^{2}, e_{d, k}=\left(\sum_{m=1}^{M} \sqrt{\vartheta_{m, k}} \Upsilon_{m, k}\right)^{2}, \tau_{u}=\left(T-\tau_{p}\right)\left(1-\alpha_{1}\right)$, $\tau_{d}=\left(T-\tau_{p}\right)\left(1-\alpha_{2}\right) \alpha_{1}, \quad \xi_{1}=\left(T-\tau_{p}\right)\left(1-\alpha_{2}\right) / T, \quad \xi_{2}=\left(T-\tau_{p}\right) / T, \quad \tau_{e}=\left(T-\tau_{p}\right) \alpha_{1} \alpha_{2}$. where $w_{s}=w_{e}=1$, which means the same priority is assigned to each goal. On this basis, formula (21) is rewritten as

$$
E_{e}=\frac{1}{F_{1}(N) / S_{e}+N P_{b t, m}}
$$

where

$$
F_{1}(N)=\left(N\left(p_{\bar{m}}+P_{c, m}\right)+\frac{1}{a} \sum_{m=1}^{M}\left(\sum_{k=1}^{K} P_{t} \vartheta_{m, k} \Upsilon_{m, k}\right)\right)
$$

According to formulas (23) and (24), when the number of servicing APs $\mathrm{N}$ is fixed, the larger $S_{e}$ is, the lower $E_{e}$ will be and the better the trade-off performance will be, so optimization is equivalent to

$$
\begin{aligned}
& \max _{\alpha_{1}} S_{e} \\
& \left\{\begin{array}{l}
c 1: \gamma_{u, k} \leqslant \frac{\eta\left(\alpha_{1} \alpha_{2}\right)}{(1-\alpha)} \psi_{k} b_{u, k}\left(c_{u, k}+d_{u, k}\right)^{-1}, \quad k=1, \cdots, K \\
c 2: \gamma_{d, k} \leqslant e_{d, k} / f_{d, k}, \quad k=1, \cdots, K \\
c 3: \sum_{k=1}^{K} \vartheta_{m, k} \leqslant P_{d}, m=1, \ldots, M \\
c 4: 0 \leqslant \alpha_{1} \leqslant 1
\end{array}\right.
\end{aligned}
$$


From the above formula, it is found that optimization is similar to the GP problem, but we note that the target is a polynomial function. If it becomes monomial, then the optimization will become a standard GP form. Since this paper involves AP selection, the parameter $\mathrm{N}$ cannot be solved by GP, and we use one-dimensional search to determine the optimal $\mathrm{N}$. the polynomial $\log _{2}(1+\gamma)$ can be approximated by the monomial $\lambda \gamma^{\mu}$ near point $\hat{\gamma}$, where $\mu=\frac{\hat{\gamma}}{\ln 2 \times(1+\hat{\gamma}) \log _{2}(1+\hat{\gamma})}$ and $\lambda=\hat{\gamma}^{-\mu} \log _{2}(1+\hat{\gamma})$; the proof is in Appendix B. Then, formula (22) can be rewritten as

$$
\begin{aligned}
S_{e} & =\xi_{1} \alpha_{1} \sum_{k=1}^{K} \lambda_{d, k} \gamma_{d, k}^{\mu_{d, k}}+\xi_{2}\left(1-\alpha_{1}\right) \sum_{k=1}^{K} \lambda_{u, k} \gamma_{u, k}^{\mu_{u, k}} \\
& \stackrel{f}{*} 2\left(\xi_{1} \alpha_{1} \xi_{2}\left(1-\alpha_{1}\right) K^{2}\left(\prod_{k=1}^{K} \lambda_{d, k} \gamma_{d, k}^{\mu_{d, k}} \prod_{k=1}^{K} \lambda_{u, k} \gamma_{u, k}^{\mu_{u, k}}\right)^{1 / K}\right) \\
& f: \sum_{k=1}^{K} x(k) \geqslant K\left(\prod_{k=1}^{K} x(k)\right)^{1 / K}
\end{aligned}
$$

Solving the above optimization problem by maximizing the minimum value $S_{e}^{\min }$ of $S_{e}$, the optimization formula is rewritten as

$$
\begin{gathered}
\max _{\alpha, \hat{\alpha}} \xi_{1} \alpha_{1} \xi_{2} \hat{\alpha} K^{2}\left(\prod_{k=1}^{K} \lambda_{d, k} \gamma_{d, k}^{\mu_{d, k}} \prod_{k=1}^{K} \lambda_{u, k} \gamma_{u, k}^{\mu_{u, k}}\right)^{1 / K} \\
\left\{\begin{array}{l}
c 1, c 2, c 3 \\
c 4: \alpha+\hat{\alpha} \leqslant 1
\end{array}\right.
\end{gathered}
$$

Formula (26) is a standard GP problem, which is solved by the algorithm in Algorithm 1.

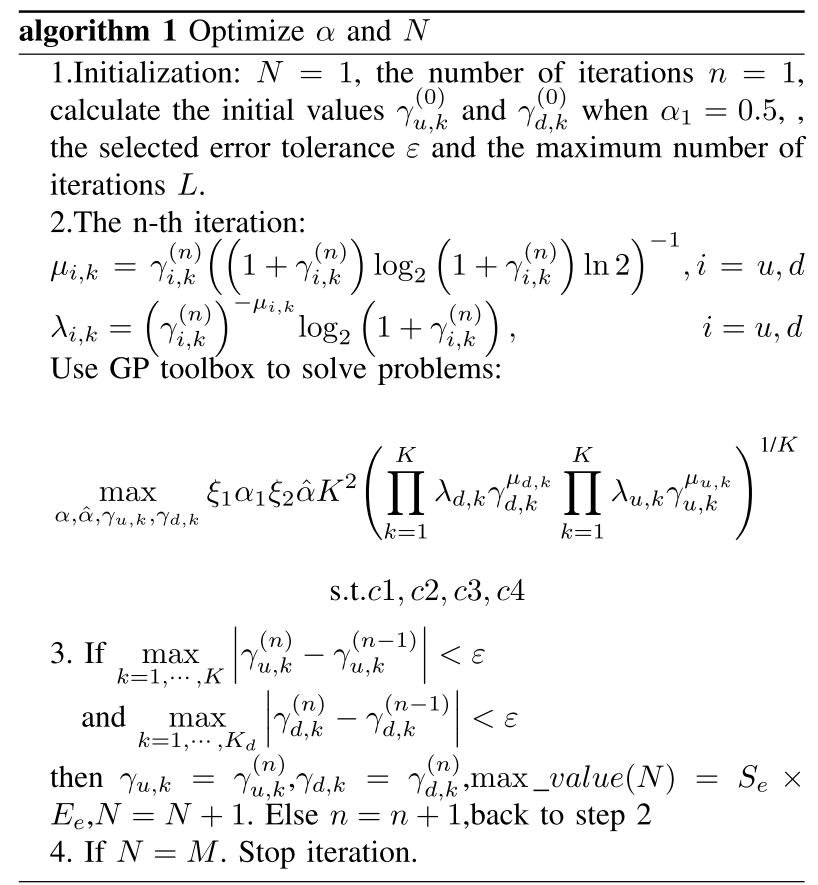


Table 1 Parameter settings

\begin{tabular}{llll}
\hline Parameter & Value & Parameter & Value \\
\hline$P_{\tau}$ & $100 \mathrm{~mW}$ & $\tau_{P}$ & 40 \\
$a$ & 0.4 & $\tau_{c}$ & 200 \\
$P_{c, m}$ & $0.2 \mathrm{~W}$ & $B_{c}$ & $100 \mathrm{kHz}$ \\
$p_{\bar{m}}$ & $0.825 \mathrm{~W}$ & $\rho_{d, l}$ & $200 \mathrm{~mW}$ \\
$\rho_{\text {total }}$ & $20 \mathrm{~W}$ & $P_{b t, m}$ & $0.25 \mathrm{~W}(\mathrm{Gbits} / \mathrm{s})$ \\
$G$ & $1.9 \mathrm{GHz}$ & $\eta$ & 0.8 \\
$B$ & $20 \mathrm{MHz}$ & $\sigma^{2}$ & $-96 \mathrm{dBm}$ \\
\hline
\end{tabular}

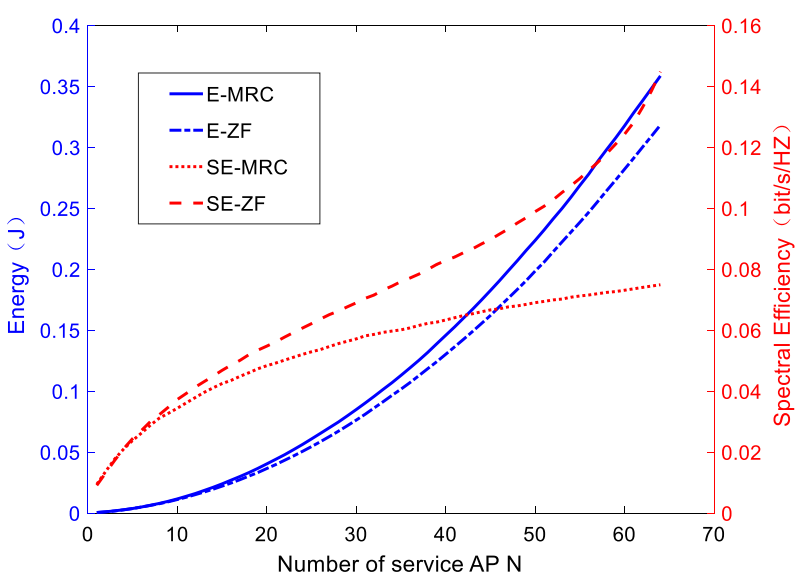

Fig. 4 Schematic diagram of the recovered energy and spectrum efficiency changing with the number of serving APs, $\tau_{e}=\tau_{d}=\frac{1}{4} T$

\section{Experiments, results and discussions}

This section shows the simulation results. This paper considers a UC CF massive MIMO SWIPT system with $M=64$ APs and $K=5$ users, which are randomly distributed in a rectangular area of $D=100 \mathrm{~m}$; the height of the antenna is $h_{A P}=15$ $\mathrm{m}$, and the height of a user is $h_{k}=1.65 \mathrm{~m}$. To simplify the calculation, each AP in this paper uses the average power allocation for the users it serves. We use the HataCOST231 propagation model for simulation in a medium-sized city and suburban model environment [41, 42]. The other simulation parameters are set as in Table 1.

Figure 4 analyses the performance in recovering the energy and SE of users with a number of antennas in the same transmission time interval when ZF precoding is used on the CPU and MRC precoding is used on the AP. This can be seen in the simulation diagram. First, due to the particularity of energy harvesting, the energy recovered when MRC precoding is used on the AP is greater than that when ZF precoding is used on the CPU. Therefore, choosing to use MRC precoding on the AP in the CF massive MIMO SWIPT system can not only reduce system complexity but also has certain advantages in energy harvesting. Second, with the increase in the number of serving APs, the energy recovered by users and the SE increase, but when $\mathrm{N}$ increases to a fixed value, the increase in SE tends to stabilize, so the AP selection strategy is 


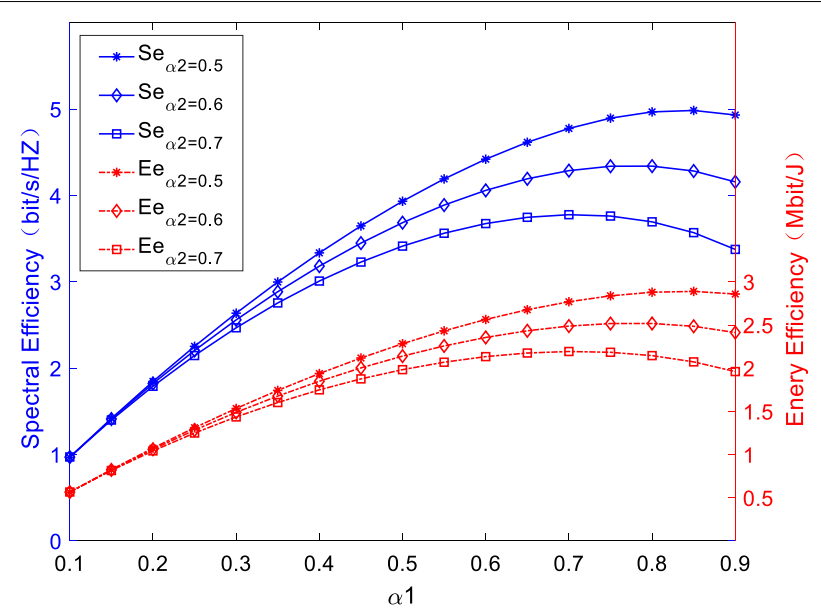

Fig. 5 Spectral efficiency and energy efficiency varying with $\alpha_{1}$ in case of $\alpha_{2}=0.5,0.6,0.7$

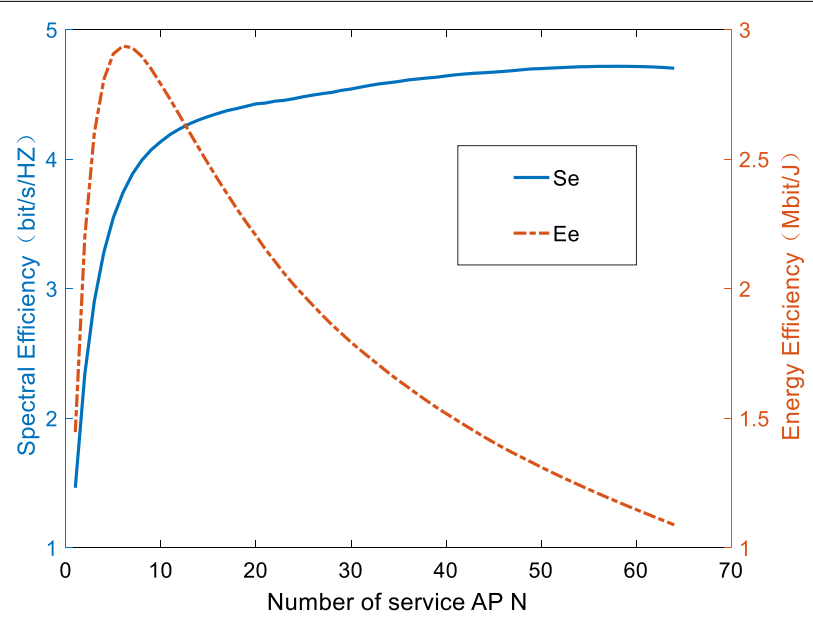

Fig. 6 Schematic diagram of system spectral efficiency and energy efficiency varying with the number of serving APs

not adopted in the energy recovery stage. In the information transmission stage, the AP selection strategy is adopted.

Figure 5 simulates the SE and EE of the system varying with the change in the DL time switching ratio $\alpha_{1}$ when the energy recovery time switching ratio $\alpha_{2}$ is fixed. It can be seen from the simulation diagram that with the increase in $\alpha_{1}$, both the SE and EE show a trend of first increasing and then decreasing, and there is a specific $\alpha_{1}$ that maximizes the system SE and EE; different $\alpha_{2}$ values correspond to different $\alpha_{1}$. When $\alpha_{2}$ is fixed, the larger $\alpha_{1}$ is, the more energy can be harvested, and the higher the power for uplink transmission for users, but the shorter the corresponding uplink user transmission time. When $\mathrm{N}$ is larger, the power provided to users is larger, and the spectral efficiency of the system is higher, but at the same time, more system energy will be consumed.

Figure 6 simulates the change in the SE and EE of the system with the change in the DL time switching ratio $\alpha_{1}$ when the energy recovery time switching ratio $\alpha_{2}$ is constant. It can be seen from the simulation diagram that with the increase in $\alpha_{1}$, 


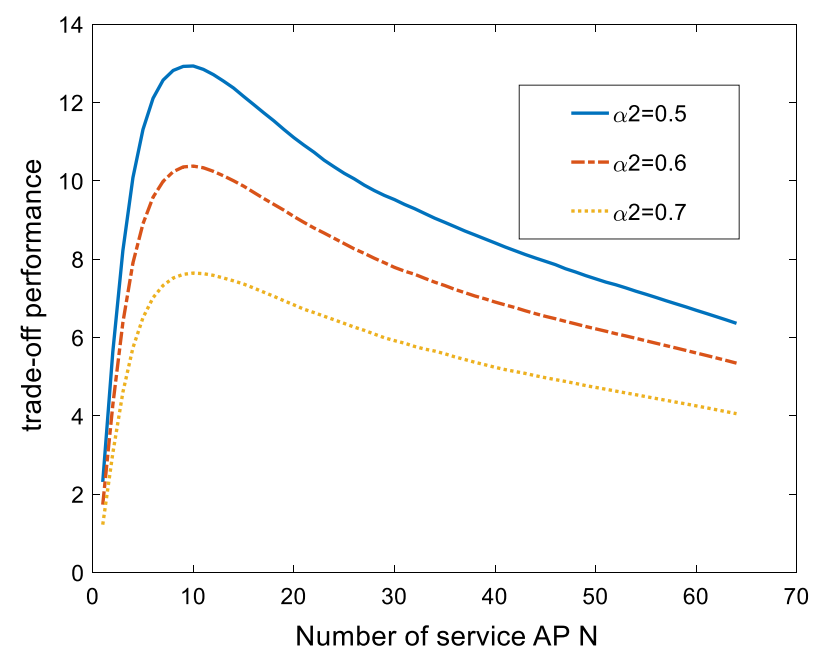

Fig. 7 System trade-off performance optimization

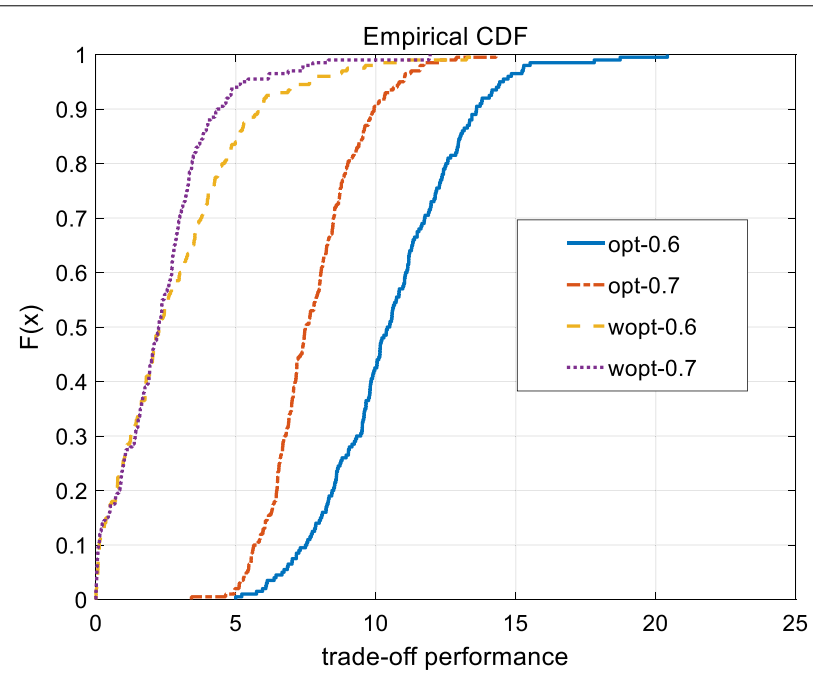

Fig. 8 Comparison of trade-off performance optimized unoptimized, $\alpha_{2}=0.6, \alpha_{2}=0.7$

both the SE and EE show a trend of first increasing and then decreasing, and there is a specific $\alpha_{1}$ that maximizes the system SE and EE; different $\alpha_{2}$ values correspond to different $\alpha_{1}$.

Figure 7 simulates and analyses the trade-off performance after optimization under different conditions of $\alpha_{2}$. The simulation results show that the smaller the value of $\alpha_{2}$ is, the better the trade-off performance of the system. This conclusion is consistent with the conclusion that the system performance is not optimized in Fig. 5, and there is an optimal number of serving APs so that the system can take into account the SE and EE.

From the simulation diagram of Fig. 8 , it can be seen that when $\alpha_{2}=0.7$, the probability of the trade-off performance trade_off $\geqslant 6$ is $95 \%$ after optimization; in the unoptimized case, the probability of trade_off $\geqslant 6$ is $5 \%$. When $\alpha_{2}=0.6$, 
the probability of trade_off $\geqslant 7$ is $95 \%$ after optimization, and the probability of trade_off $\geqslant 7$ is $10 \%$ without optimization. Therefore, the simulation shows the superiority of the optimized scheme.

Massive MIMO increases the number of antennas and serves more users within the same time-frequency resources. The BS can obtain multiplexing gain and array gain at the same time [3], but according to the formula (20), increasing the number of antennas will result in a decrease in energy efficiency. The local MRC precoding method proposed in this article is that the AP only needs to know the estimated channel state information of the serving user, but compared to the centralized ZF precoding method, the local MRC spectral efficiency is lower. This paper is to strike a balance between spectral efficiency and energy efficiency, sacrificing part of the spectral efficiency in exchange for an improvement in energy efficiency.

Figures 9 and 10 compare the SE and EE of the Two schemes using MRC (optimized and not optimized) and ZF (local processing [43] and global processing). When simulating the unoptimized MRC scheme and ZF scheme, the UL and DL time switching ratio $\alpha_{1}$ is randomly generated. It can be seen from Fig. 9 that the scheme proposed in this article has no obvious advantage in terms of SE, this is because, firstly the MRC/MRT method ignores multi-user interference, and its performance is not as good as ZF [1], secondly due to AP selection and an optimization solution that takes EE into account, there is a slight loss in SE. However, the computational complexity of ZF is $O\left(M+K^{3}\right)$, while MRC is $O(M)$. Figure 10 reflects the advantages of the optimization scheme in this paper. The optimized EE is not only twice of the unoptimized EE but also exceeds the local zero-forcing and is infinitely close to the global zero-forcing. but in each coherence block, Number of complex scalars send from the APs to the CPU via the fronthaul in global processing is more $T M$ than local processing. The simulation results show that the optimization method proposed in this paper can reduce the information interaction between CPU and AP, and improve energy efficiency.

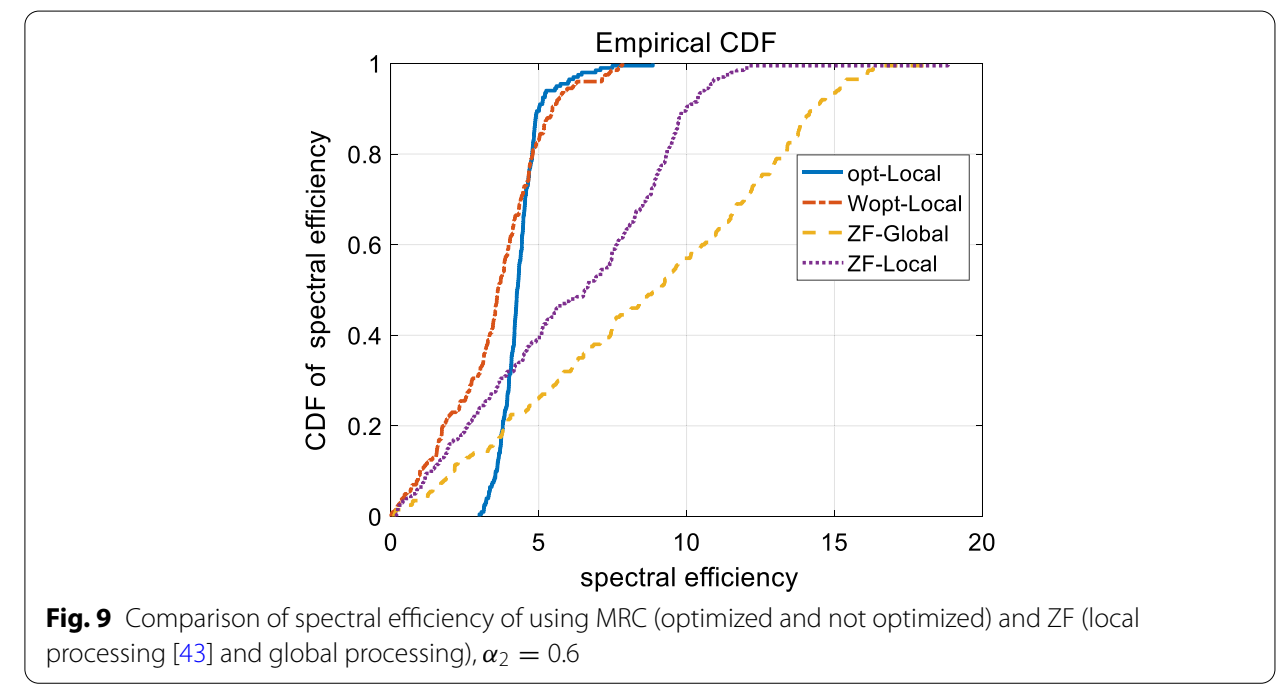




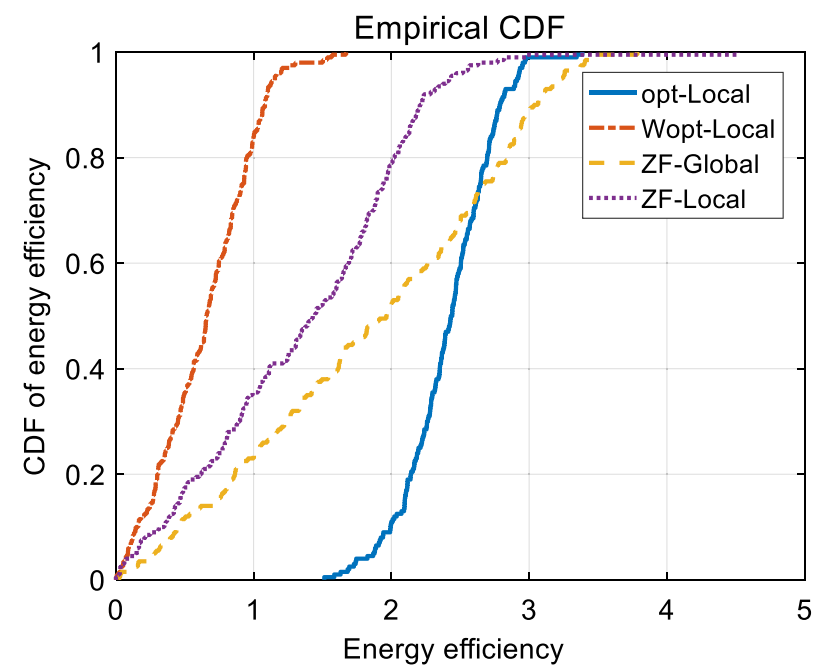

Fig. 10 Comparison of energy efficiency of using MRC (optimized and not optimized) and ZF (local processing [43] and global processing), $\alpha_{2}=0.6$

\section{Conclusion}

In this work, we study a UC CF massive MIMO SWIPT system. SWIPT technology in a centralized massive MIMO system is greatly affected by the large scale due to its distance from the BS, resulting in very little harvested energy, and a distributed antenna can just make up for this defect. According to the characteristics of distributed antennas, some APs are far from specific users, and AP selection schemes based on channel strength are proposed. The transmission process for each frame is divided into four parts: channel estimation, DL energy transmission, DL information transmission, and the use of recovered energy for UL information transmission. EE is a focus of close attention in 6G. This article proposes a trade-off index that takes into account the system SE and EE. The AP selection scheme and the UL and DL time switching ratio are jointly optimized to maximize the trade-off performance of the system and solve the non-convex problem. The non-convex problem is transformed into a GP problem to be solved. The simulation results show that the optimized scheme achieves better EE performance, and the trade-off performance of the system is the strongest. In addition, this solution can reduce the load of the CPU and reduce the system complexity.

\section{Appendix A}

$$
\begin{aligned}
\mathrm{F}_{1} & =\sum_{m=1}^{M} \mathbb{E}\left\{g_{m, k} \sqrt{\vartheta_{m, k} P_{t}} \alpha_{m} \hat{g}_{m, k}^{H}\right\} \\
& =\sum_{m=1}^{M} \mathbb{E}\left\{\left(\hat{g}_{m, k}+e\right) \sqrt{\vartheta_{m, k} P_{t}} \alpha_{m} \hat{g}_{m, k}^{H}\right\} \\
& =\sum_{m=1}^{M} \alpha_{m} \sqrt{\vartheta_{m, k} P_{t}} \mathbb{E}\left\{\left|\hat{g}_{m, k}\right|^{2}\right\} \\
& =\sum_{m=1}^{M} \alpha_{m} \sqrt{\vartheta_{m, k} P_{t}} \Upsilon_{m, k}
\end{aligned}
$$




$$
\begin{aligned}
& \mathrm{F}_{2}=P_{t} \sum_{m=1}^{M} \vartheta_{m, k} \mathbb{E}\left\{\left|g_{m, k} \alpha_{m} \hat{g}_{m, k}^{H}-\mathbb{E}\left\{g_{m, k} \alpha_{m} \hat{g}_{m, k}^{H}\right\}\right|^{2}\right\} \\
& =P_{t} \sum_{m=1}^{M} \alpha_{m}^{2} \vartheta_{m, k}\left(\mathbb{E}\left\{\left|g_{m, k} \hat{g}_{m, k}^{H}\right|^{2}\right\}-\left|\mathbb{E}\left\{g_{m, k} \hat{g}_{m, k}^{H}\right\}\right|^{2}\right) \\
& =P_{t} \sum_{m=1}^{M} \alpha_{m}^{2} \vartheta_{m, k}\left(\mathbb{E}\left\{\left|g_{m, k}^{H} \hat{g}_{m, k}^{H}\right|^{2}\right\}+\mathbb{E}\left\{\left|e \hat{g}_{m, k}^{H}\right|^{2}\right\}-\Upsilon_{m, k}^{2}\right) \\
& =P_{t} \sum_{m=1}^{M} \alpha_{m}^{2} \vartheta_{m, k}\left(2 \Upsilon_{m, k}^{2}+\Upsilon_{m, k}\left(\beta_{m, k}-\Upsilon_{m, k}\right)-\Upsilon_{m, k}^{2}\right) \\
& =P_{t} \sum_{m=1}^{M} \alpha_{m}^{2} \vartheta_{m, k} \beta_{m, k} \Upsilon_{m, k} \\
& \mathrm{~F}_{3}=\mathbb{E}\left\{P_{t}\left|\sum_{m=1}^{M} g_{m, k} \vartheta_{m, k^{\prime}}^{1 / 2} \alpha_{m} c_{m, k^{\prime}}\left(\sqrt{\tau_{p} P_{p}} g_{m, k^{\prime}}+\mathbf{N}_{m} \mathbf{s}_{k^{\prime}}^{H}\right)^{H}\right|^{2}\right\} \\
& =\underbrace{\mathbb{E}\left\{\left|\sum_{m=1}^{M} g_{m, k} \sqrt{\vartheta_{m, k^{\prime}} P_{t}} \alpha_{m} c_{m, k^{\prime}} \sqrt{\tau_{p} P_{p}} g_{m, k^{\prime}}^{H}\right|^{2}\right\}}_{\mathrm{F}_{31}} \\
& +\underbrace{\mathbb{E}\left\{\left|\sum_{m=1}^{M} g_{m, k} \sqrt{\vartheta_{m, k^{\prime}} P_{t}} \alpha_{m} c_{m, k^{\prime}} \mathbf{N}_{m}^{H}\right|^{2}\right\}}_{\mathrm{F}_{32}}
\end{aligned}
$$

where

$$
\begin{aligned}
\mathrm{F}_{31} & =\tau_{p} P_{p} P_{t} \mathbb{E}\left\{\left|\sum_{m=1}^{M} \sqrt{\vartheta_{m, k^{\prime}}} \alpha_{m} c_{m, k^{\prime}} g_{m, k} g_{m, k^{\prime}}^{H}\right|^{2}\right\} \\
& =\tau_{p} P_{p} P_{t} \sum_{m=1}^{M} \alpha_{m}^{2} \vartheta_{m, k^{\prime}} c_{m, k^{\prime}}^{2} \beta_{m, k} \beta_{m, k^{\prime}} \\
\mathrm{F}_{32} & =P_{t} \sum_{m=1}^{M} \beta_{m, k} \alpha_{m}^{2} \vartheta_{m, k^{\prime}} c_{m, k^{\prime}}^{2} \sigma_{2} \\
\mathrm{~F}_{3} & =\mathrm{F}_{31}+\mathrm{F}_{32}=P_{t} \sum_{m=1}^{M} \alpha_{m}^{2} \vartheta_{m, k} \beta_{m, k} \Upsilon_{m, k} \\
& +\sum_{k^{\prime} \in \kappa(m), k^{\prime} \neq k}\left(\sum_{m=1}^{M} P_{t} \alpha_{m}^{2} \vartheta_{m, k^{\prime}} \Upsilon_{m, k^{\prime}}^{2} \beta_{m, k}\right)+\sigma^{2} \\
& =\sum_{k^{\prime} \in \kappa(m)}\left(\sum_{m=1}^{M} P_{t} \alpha_{m}^{2} \vartheta_{m, k^{\prime}} \Upsilon_{m, k^{\prime}}^{2} \beta_{m, k}\right)+\sigma^{2}
\end{aligned}
$$




\section{Appendix B: Proof of Lemma 1}

set

$$
y_{1}=\lambda \gamma^{\mu}, y_{2}=\log _{2}(1+\gamma)
$$

then

$$
y_{1}(\hat{\gamma})=y_{2}(\hat{\gamma}), y_{1}^{\prime}(\hat{\gamma})=y_{2}^{\prime}(\hat{\gamma})
$$

then

$$
\left\{\begin{array}{l}
\lambda \hat{\gamma}^{\mu}=\log _{2}(1+\gamma) \\
\lambda \mu \hat{\gamma}^{\mu-1}=\frac{1}{\ln 2 \times(1+\gamma)}
\end{array}\right.
$$

so

$$
\mu=\frac{\hat{\gamma}}{\ln 2 \times(1+\hat{\gamma}) \log _{2}(1+\hat{\gamma})}, \lambda=\hat{\gamma}^{-\mu} \log _{2}(1+\hat{\gamma})
$$

\section{Abbreviations}

AP: Access point; CF: Cell-free; MIMO: Multi-input multi-output; CPU: Central processing unit; DL: Downlink; EE: Energy efficiency; GP: Geometric planning; SE: Spectral efficiency; SWIPT: Simultaneous wireless information and power transmission; UC: User-centric; UL: Uplink.

\section{Acknowledgements}

Not applicable

\section{Authors' contributions}

LN proposes the innovation ideas and carries out experiments and data analysis. GYY and XK reviewed the manuscript and fixed some issues. All authors read and approved the final manuscript.

\section{Funding}

This research was funded by the National Natural Science Foundation of China, Grant No. 61501511.

Availability of data and materials

Not applicable.

\section{Declarations}

\section{Ethics approval and consent to participate}

Not applicable.

\section{Consent for publication}

Not applicable

\section{Competing interests}

The authors declare that they have no competing interests.

Received: 4 February 2021 Accepted: 27 July 2021

Published online: 17 August 2021

\section{References}

1. E. Bjornson, J. Hoydis, L. Sanguinetti, Massive MIMO networks: spectral, energy, and hardware efficiency. Found. Trends R Signal Process. 11(3-4), 154-655 (2017)

2. E.G. Larsson, O. Edfors, F. Tufvesson et al., Massive MIMO for next generation wireless systems. Commun. Mag. 52(2), 186-195 (2014)

3. H.Q. Ngo, E.G. Larsson, T.L. Marzetta, Energy and spectral efficiency of very large multiuser MIMO systems. IEEE Trans. Commun. 61(4), 1436-1449 (2013). https://doi.org/10.1109/TCOMM.2013.020413.110848

4. H.Q. Ngo, A. Ashikhmin, H. Yang, E.G. Larsson, T.L. Marzetta, Cell-free massive MIMO: uniformly great service for everyone. in Poc. SPAWC, pp. 201-205 (2015) 
5. S. Timilsina, D. Kudathanthirige, G. Amarasuriya, Physical layer security in cell-free massive MIMO, in Proc. GLOBECOM, AbuDhabi, United Arab Emirates, pp. 1-7 (2018). https://doi.org/10.1109/GLOCOM.2018.8647876

6. G. Interdonato, H.Q. Ngo, P. Frenger, E.G. Larsson, Downlink training in cell-free massive MIMO: a blessing in disguise. IEEE Trans. Wirel. Commun. 18(11), 5153-5169 (2019). https://doi.org/10.1109/TWC.2019.2933831

7. O. Ozdogan, E. Bjornson, J. Zhang, Performance of cell-free massive MIMO with Rician fading and phase shifts. IEEE Trans. Wirel. Commun. 18(11), 5299-5315 (2019). https://doi.org/10.1109/TWC.2019.2935434

8. M. Bashar, H.Q. Ngo, A.G. Burr, D. Maryopi, K. Cumanan, E.G. Larsson, On the performance of backhaul constrained cell-freemassive MIMO with linear receivers. in 2018 52nd Asilomar Conference on Signals, Systems, and Computers, Pacific Grove, CA,USA, pp. 624-628 (2018). https://doi.org/10.1109/ACSSC.2018.8645433

9. Z. Lin, M. Lin, B. Champagne, W.-P. Zhu, N. Al-Dhahir, Secure and energy efficient transmission for RSMA-based cognitivesatellite-terrestrial networks. IEEE Wirel. Commun. Lett. 10(2), 251-255 (2021)

10. S. Buzzi, C. D'Andrea, Cell-free massive MIMO: user-centric approach. IEEE Wirel. Commun. Lett. 6(6), 706-709 (2017). https://doi.org/10.1109/LWC.2017.2734893

11. Z. Lin, M. Lin, T. de Cola, J.B. Wang, W.P. Zhu, J. Cheng, Supporting loT with rate-splitting multiple access in satellite and aerial integrated networks. IEEE Internet Things J. (2021). https://doi.org/10.1109/JIOT.2021.3051603

12. X. Wu, W. Xu, X. Dong, H. Zhang, X. You, Asymptotically optimal power allocation for massive MIMO wireless powered communications. IEEE Wirel. Commun. Lett. 5(1), 100-103 (2016). https://doi.org/10.1109/LWC.2015.2502939

13. S. Ulukus et al., Energy harvesting wireless communications: a review of recent advances. IEEE J. Sel. Areas Commun. 33(3), 360-381 (2015). https://doi.org/10.1109/JSAC.2015.2391531

14. K. Xu, Z. Shen, Y. Wang, X. Xia, D. Zhang, Hybrid time-switching and power splitting SWIPT for full-duplex massive MIMO systems: a beam-domain approach. IEEE Trans. Veh. Technol. 67(8), 7257-7274 (2018). https://doi.org/10. 1109/TVT.2018.2831790

15. Z. Zhu, Z. Chu, F. Zhou, H. Niu, Z. Wang, I. Lee, Secure beamforming designs for secrecy MIMO SWIPT systems. IEEE Wirel. Commun. Lett. 7(3), 424-427 (2018). https://doi.org/10.1109/LWC.2017.2780830

16. L.R. Varshney, Transporting information and energy simultaneously, in IEEE International Symposium on Information Theory. Toronto, ON, pp. 1612-1616 (2008)

17. R. Zhang, C.K. Ho, MIMO broadcasting for simultaneous wireless information and power transfer. IEEE Trans. Wirel. Commun. 12(5), 1989-2001 (2013)

18. S. Buzzi, C. D'Andrea, User-centric communications versus cell-free massive MIMO for $5 G$ cellular networks. in WSA 2017; 21th International ITG Workshop on Smart Antennas, Berlin, Germany, pp. 1-6 (2017)

19. J. Hoydis, S. ten Brink, M. Debbah, Massive MIMO in the UL/DL of cellular networks: how many antennas do we need? IEEE J. Sel. Areas Commun. 31(2), 160-171 (2013). https://doi.org/10.1109/JSAC.2013.130205

20. S. Jin, X. Liang, K. Wong, X. Gao, Q. Zhu, Ergodic rate analysis for multipair massive MIMO two-way relay networks. IEEE Trans. Wirel. Commun. 14(3), 1480-1491 (2015). https://doi.org/10.1109/TWC.2014.2367503

21. F. Sohrabi, W. Yu, Hybrid digital and analog beamforming design for large-scale antenna arrays. IEEE J. Sel. Top. Signal Process. 10(3), 501-513 (2016). https://doi.org/10.1109/JSTSP.2016.2520912

22. T. Van Chien, E. Bjornson, E.G. Larsson, Joint power allocation and user association optimization for massive MIMO systems. IEEE Trans. Wirel. Commun. 15(9), 6384-6399 (2016). https://doi.org/10.1109/TWC.2016.2583436

23. M. Bashar, K. Cumanan, A.G. Burr, H.Q. Ngo, M. Debbah, Cell-free massive MIMO with limited backhaul, in 2018 IEEEInternational Conference on Communications (ICC), Kansas City, MO, pp. 1-7 (2018). https://doi.org/10.1109/ICC.2018. 8422865

24. Z. Gao, L. Dai, D. Mi, Z. Wang, M.A. Imran, M.Z. Shakir, MmWave massive-MIMO-based wireless backhaul for the 5 G ultra-dense network. IEEE Wirel. Commun. 22(5), 13-21 (2015). https://doi.org/10.1109/MWC.2015.7306533

25. R. Mahapatra, Y. Nijsure, G. Kaddoum, N. UI Hassan, C. Yuen, Energy efficiency tradeoff mechanism towards wireless green communication: a survey. IEEE Commun. Surv. Tutor. 18(1), 686-705 (2016). https://doi.org/10.1109/COMST. 2015.2490540

26. Z. Lin, M. Lin, B. Champagne, W.-P. Zhu, N. Al-Dhahir, Secure beamforming for cognitive satellite terrestrial networks with unknown eavesdroppers. IEEE Syst. J. (2020). https://doi.org/10.1109/JSYST.2020.2983309

27. Z. Lin, M. Lin, B. Champagne, W.-P. Zhu, N. Al-Dhahir, Secrecy-energy efficient hybrid beamforming for satelliteterrestrial integrated networks. IEEE Trans. Commun

28. F. Heliot, O. Onireti, M.A. Imran, An accurate closed-form approximation of the energy efficiency-spectral efficiency trade-off over the MIMO Rayleigh fading channel. in 2011 IEEE International Conference on Communications Workshops (ICC), Kyoto (2011)

29. H.Q. Ngo, L. Tran, T.Q. Duong, M. Matthaiou, E.G. Larsson, On the total energy efficiency of cell-free massive MIMO. IEEE Trans. Green Commun. Netw. 2(1), 25-39 (2018). https://doi.org/10.1109/TGCN.2017.2770215

30. L. Fan, H. Zhang, Y. Huang, L.Y. ang, Exploiting BS antenna tilt forSWIPT in 3-D Massive MIMO systems. IEEE Wirel. Commun. Lett. 6(5), 666-669 (2017)

31. Z. Chang, Z. Wang, X. Guo, Z. Han, T. Ristaniemi, Energy-efficient resource allocation for wireless powered massive MIMO system with imperfect CSI. IEEE Trans. Green Commun. Netw. 1(2), 121-130 (2017)

32. S. Mukherjee, S.K. Mohammed, Energy-spectral efficiency trade-off for a massive SU-MIMO system with transceiver power consumption. IEEE Int. Conf. Commun. (ICC) 2015, 1938-1944 (2015). https://doi.org/10.1109/ICC.2015. 7248609

33. P. Patcharamaneepakorn et al., Spectral, energy, and economic efficiency of $5 \mathrm{G}$ multicell massive $\mathrm{MIMO}$ systems with generalized spatial modulation. IEEE Trans. Veh. Technol. 65(12), 9715-9731 (2016). https://doi.org/10.1109/TVT. 2016.2526628

34. D. Kudathanthirige, R. Shrestha, G.A. Aruma Baduge, Max-Min fairness optimal rate-energy trade-off of SWIPT for massive MIMO downlink. IEEE Commun. Lett. 23(4), 688-691 (2019). https://doi.org/10.1109/LCOMM.2019.2896609

35. J. Xiong, L. You, A. Zappone, W. Wang, X. Gao, Energy- and spectral-efficiency tradeoff in beam domain massive MIMO Downlink with Statistical CSIT. in IEEE Global Conference on Signal and Information Processing (GlobalSIP), vol. 2019, pp. 1-5 (2019). https://doi.org/10.1109/GlobalSIP45357.2019.8969338 
36. M.Y. Ali, T. Hossain, M.M. Mowla, A trade-off between energy and spectral efficiency in massive MIMO 5G system. in 20193 rd International Conference on Electrical, Computer and Telecommunication Engineering (ICECTE), pp. 209-212 (2019). https://doi.org/10.1109//CECTE48615.2019.9303551

37. R. Shrestha, G. Amarasuriya, SWIPT in cell-free massive MIMO. in 2018 IEEE Global Communications Conference (GLOBECOM), Abu Dhabi, United Arab Emirates, pp. 1-7 (2018). https://doi.org/10.1109/GLOCOM.2018.8648129

38. S. Kusaladharma, W. Zhu, W. Ajib, G. Amarasuriya, Performance of SWIPT in cell-free massive MIMO: a stochastic geometry based perspective. in 2020 IEEE 17th Annual Consumer Communications and Networking Conference (CCNC), Las Vegas, NV, USA, pp. 1-6 (2020). https://doi.org/10.1109/CCNC46108.2020.9045726

39. F. Tan, P. Wu, Y. Wu, M. Xia, Energy-efficient non-orthogonal multicast and unicast transmission of cell-free massive MIMO systems with SWIPT. IEEE J. Sel. Areas Commun. (2020). https://doi.org/10.1109/JSAC.2020.3020110

40. T.L. Marzetta, E.G. Larsson, H.Y. ang, H.Q. Ngo, Fundamentals of Massive MIMO (Cambridge University Press, Cambridge, 2016)

41. H.Q. Ngo, A. Ashikhmin, H. Yang, E.G. Larsson, T.L. Marzetta, Cell-free massive MIMO versus small cells. IEEE Trans. Wirel. Commun. 16(3), 1834-1850 (2017). https://doi.org/10.1109/TWC.2017.2655515

42. B.S.L. Castro, I.R. Gomes, F.C.J. Ribeiro, G.P.S. Cavalcante, COST231-Hata and SUI models performance using a LMS tuning algorithm on $5.8 \mathrm{GHz}$ in Amazon Region cities. in Proceedings of the Fourth European Conference on Antennas and Propagation, pp. 1-3 (2010)

43. G. Interdonato, M. Karlsson, E. Bjornson, E.G. Larsson, Local partial zero-forcing precoding for cell-free massive MIMO, IEEE Trans. Wirel. Commun. 19(7), 4758-4774 (2020). https://doi.org/10.1109/TWC.2020.2987027

\section{Publisher's Note}

Springer Nature remains neutral with regard to jurisdictional claims in published maps and institutional affiliations.

\section{Submit your manuscript to a SpringerOpen ${ }^{\circ}$ journal and benefit from:}

- Convenient online submission

Rigorous peer review

- Open access: articles freely available online

- High visibility within the field

- Retaining the copyright to your article

Submit your next manuscript at $\mathbf{s p r i n g e r o p e n . c o m ~}$ 